\title{
Second hit influences the interaction between tumor cells and the immune system in a murine model of Burkitt's lymphoma Christian Schuster ${ }^{1}$, Anna Frenzel ${ }^{2}$, Andrea Hölbl ${ }^{1}$, Olivia Simma1, Mathias Müller ${ }^{3}$, Andreas Villunger ${ }^{2}$, Veronika Sexl ${ }^{1}$ and Dagmar Stoiber*1,4
}

\author{
Address: ${ }^{1}$ Department of Pharmacology, Medical University of Vienna, 1090 Vienna, Austria, ${ }^{2}$ Division of Developmental Immunology, Medical \\ University of Innsbruck, 6020, Austria, ${ }^{3}$ Institute of Animal Breeding and Genetics, Veterinary University of Vienna, and Austrian Center for \\ Biomodels and Transgenetics (ÖZBT VUW), 1220 Vienna, Austria and ${ }^{4}$ Ludwig Boltzmann Institute for Cancer Research, 1090 Vienna, Austria \\ Email: Dagmar Stoiber* - dagmar.stoiber@meduniwien.ac.at \\ * Corresponding author
}

from 14th Scientific Symposium of the Austrian Pharmacological Society (APHAR)

Innsbruck, Austria. 21-22 November 2008

Published: 5 November 2008

BMC Pharmacology 2008, 8(Suppl I):A2I doi:I0.II86/I47I-22I0-8-SI-A2I

This abstract is available from: http://www.biomedcentral.com/I47I-22I0/8/SI/A2I

(C) 2008 Schuster et al; licensee BioMed Central Ltd.

The process of cancerogenesis is driven by the hierarchical accumulation of genetic changes. So, beside the deregulated expression of an oncogen, additional genetic defects have to take place in order to transform a normal cell into a tumor cell. In case of Burkitt's lymphoma, a c-mycdriven haematological tumor, two predominant so called 2nd hits have been identified. First, loss-of-function mutations in the p19-Mdm2-p53 tumor suppressor pathway, and second, the over-expression of the anti-apoptotic protein Bcl-2. So far it is not known whether these two different 2 nd hits translate into cell-autonomous effects or the ability of the immune system of recognizing and destroying the tumor cells. Our studies revealed that neither over-expression of Bcl-2 nor loss-of-function mutations in the p19-Mdm2-p53 tumor suppressor pathway alters any analysed cell-autonomous effect significantly. However, we could specify an impact of these additional genetic lesions on the interaction between immune system and tumor cells. We were able to show that tumor cells over-expressing $\mathrm{Bcl}-2$ can be eliminated by the immune system more effectively than ones with p19Mdm2-p53 tumor suppressor pathway malfunctions. These data are in line with the analysis regarding 2 nd hits of primary tumor samples obtained form an animal model of Burkitt's lymphoma. This analysis showed a clear survival advantage of mice suffering from tumors over-expressing Bcl-2. Taken together, our studies provide basic knowledge concerning the influence of the 2 nd hit on the interaction between immune system and tumor cells, which might have prognostic value for immune based cancer therapies. 